\title{
Competencias digitales en profesores de educación superior de Iberoamérica: una revisión sistemática
}

Digital Competences in Higher Education Teachers in Iberoamerica: A systematic review

\section{Competências digitais em professores de ensino superior na Ibero-América: uma revisão sistemática}

Juan Carlos Padilla Escobedo

Universidad de Guadalajara, México

jcpadillae@gmail.com http://orcid.org/0000-0002-7207-2404

Graciela Gerarda Ayala Jiménez Universidad Autónoma de Querétaro, México

chela_aj@hotmail.com http://orcid.org/0000-0002-1889-1804

\section{Resumen}

El objetivo del presente trabajo fue realizar una revisión literaria en repositorios de documentos científicos sobre el tema competencias digitales de docentes universitarios de Iberoamérica. A partir de criterios de búsqueda establecidos, se encontraron en las bases de datos consultadas más de ocho mil artículos. Sin embargo, se eligieron únicamente aquellos con menos de cinco años de antigüedad, de corte empírico y escritos en español. Los artículos analizados (19 en total) mostraron que la mayor producción sobre el tema en Iberoamérica se ubica en España en comparación con el entorno latinoamericano. Asimismo, se concluye que los estudios se han enfocado más en el componente tecnológico de las TIC que en los aspectos de índole pedagógica. Por ello, cabe resaltar que el simple empleo de las TIC en el proceso de enseñanza-aprendizaje es insuficiente para fomentar cambios radicales en la educación formal, ya que lo más significativo es habilitar a los 


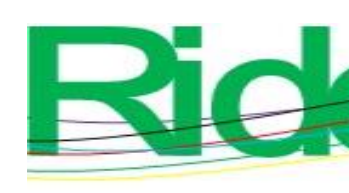
Revista Iberoamericana para la
Investigación y el Desarrollo Educativo
ISSN $2007-7467$

docentes en el empleo de competencias digitales que potencien el aprendizaje real de los estudiantes.

Palabras clave: competencia digital, docente, educación superior, revisión sistemática, tecnologías de la información y la comunicación.

\begin{abstract}
The objective of this work was to carry out a literary review in repositories of scientific documents on the subject of digital competences of university teachers in Ibero-America. Based on established search criteria, more than eight thousand articles were found in the databases consulted. However, only those with less than five years old, empirical and written in Spanish were chosen. The articles analyzed (19 in total) showed that the highest production on the subject in Latin America is located in Spain compared to the Latin American environment. Likewise, it is concluded that the studies have focused more on the technological component of ICT than on aspects of a pedagogical nature. Therefore, it should be noted that the simple use of ICT in the teaching-learning process is insufficient to promote radical changes in formal education, since the most significant thing is to enable teachers to use digital skills that enhance learning real students.
\end{abstract}

Keywords: digital competence, teaching, higher education, systematic review, information and communication technologies.

\title{
Resumo
}

O objetivo deste trabalho foi realizar uma revisão literária em repositórios de documentos científicos sobre a temática das competências digitais de professores universitários da IberoAmérica. Com base nos critérios de busca estabelecidos, foram encontrados mais de oito mil artigos nas bases de dados consultadas. No entanto, foram escolhidos apenas aqueles com menos de cinco anos, empíricos e escritos em espanhol. Os artigos analisados (19 no total) mostraram que a maior produção sobre o assunto na América Latina está localizada na Espanha em comparação com o ambiente latino-americano. Da mesma forma, conclui-se que os estudos se concentraram mais na componente tecnológica das TIC do que em aspectos de natureza pedagógica. Portanto, deve-se destacar que a simples utilização das TIC no processo de ensino-aprendizagem é insuficiente para promover mudanças radicais na educação formal, já que o mais significativo é capacitar os professores a utilizarem competências digitais que potencializem a aprendizagem de alunos reais. 


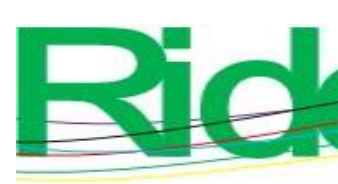

Revista Iberoamericana para la Investigación y el Desarrollo Educativo ISSN $2007-7467$

empírico, por lo que se descartaron aquellas que realizaban reflexiones sobre el tema, diseño de instrumentos u otro tipo de ensayos.

\section{Resultados}

Como ya se señaló, se utilizó digital competence como palabra clave de búsqueda en cada una de las bases de datos. En principio, se encontraron 5089 artículos en Scopus, 2447 en Web of Science (WOS) y 609 en Redalyc, de los cuales fueron seleccionados solo los escritos en español. Al respecto, valga acotar que se hallaron diversos metanálisis sobre el tema competencia digital (Delgado, Vázquez-Cano, Belando y López, 2019; Hernández, Gisbert y Fernández, 2018; Perdomo, González y Barrutia, 2020; Rodríguez y Martínez, 2018; Reiss, Pessoa y GallegoArrufat, 2019; Rodríguez, Raso y Ruiz, 2019; Rodríguez, Trujillo y Sánchez, 2019).

Posteriormente, en una búsqueda más selectiva, se advirtió que en español también existían artículos enfocados en la competencia digital de otros actores distintos a los docentes; por ejemplo, estudiantes universitarios (Castellanos, Sánchez y Calderero, 2017; Gutiérrez y Cabero, 2016; Vázquez-Cano, Reyes, Colmenares y López, 2017), así como de primaria, secundaria y educación especial. Incluso, trabajos que analizaban la influencia del entorno familiar en el desarrollo de dichas competencias (Martínez, Vila y Gewerc, 2019; Sánchez, Andrés y Paredes, 2019) o desde un enfoque cualitativo (Padilla, Gámiz y Romero, 2020).

Asimismo, en estas primeras búsquedas, se detectó otro cúmulo de indagaciones sobre la competencia digital docente, pero fuera del contexto de la educación superior (Falcó, 2017; Muñoz y Cubo, 2019; Pérez y Rodríguez, 2016; Pozo, López, Fernández y López, 2020; Roblizo, Sánchez y Cózar, 2015).

Finalmente, luego de descartar los trabajos anteriores, y considerando solo los criterios descritos en el apartado metodológico, se encontraron 19 artículos enfocados en las competencias digitales de profesores de nivel universitario a nivel de Iberoamérica. A estas publicaciones se les realizó una segmentación por contexto y año. En la tabla 1 se muestran los artículos encontrados según los criterios de búsqueda referidos. 


\begin{tabular}{|c|c|c|c|c|}
\hline $\begin{array}{l}\text { Solís de Ovando y } \\
\text { Jara }\end{array}$ & Chile & 2019 & $\begin{array}{l}\text { Competencia digital de docentes de } \\
\text { ciencias de la salud de una } \\
\text { universidad chilena }\end{array}$ & Observacional \\
\hline $\begin{array}{l}\text { Ríos, Gómez y } \\
\text { Rojas }\end{array}$ & Chile & 2018 & $\begin{array}{l}\text { Valoración de competencias TIC } \\
\text { del profesorado universitario: un } \\
\text { caso en Chile }\end{array}$ & Observacional \\
\hline Pérez & $\begin{array}{l}\text { República } \\
\text { Dominicana }\end{array}$ & 2019 & $\begin{array}{l}\text { Competencia digital docente en los } \\
\text { institutos superiores de formación } \\
\text { de maestros: caso de República } \\
\text { Dominicana }\end{array}$ & Observacional \\
\hline $\begin{array}{l}\text { Sandia, Aguilar y } \\
\text { Luzardo }\end{array}$ & Venezuela & 2018 & $\begin{array}{l}\text { Competencias digitales de los } \\
\text { docentes de educación superior. } \\
\text { Caso Universidad de Los Andes }\end{array}$ & Observacional \\
\hline $\begin{array}{l}\text { Zambrano, } \\
\text { Marquina, Araque } \\
\text { y Mousalli }\end{array}$ & Venezuela & 2016 & $\begin{array}{l}\text { Escuela de educación y la } \\
\text { competencia digital de sus } \\
\text { estudiantes y docentes }\end{array}$ & Observacional \\
\hline $\begin{array}{l}\text { Avello, López y } \\
\text { Vázquez }\end{array}$ & Cuba & 2016 & $\begin{array}{l}\text { Competencias TIC de los docentes } \\
\text { de las escuelas de Hotelería y } \\
\text { Turismo cubanas }\end{array}$ & Observacional \\
\hline $\begin{array}{l}\text { Zárate, Gurieva y } \\
\text { Jiménez }\end{array}$ & México & 2020 & $\begin{array}{l}\text { La práctica holística de las } \\
\text { competencias digitales docentes: } \\
\text { diagnóstico y prospectiva }\end{array}$ & Observacional \\
\hline Araiza y Pedraza & México & 2019 & $\begin{array}{l}\text { Discernimiento de los docentes por } \\
\text { género en el uso de las TIC en el } \\
\text { aula a partir de las competencias } \\
\text { digitales }\end{array}$ & Observacional \\
\hline $\begin{array}{l}\text { Zempoalteca, } \\
\text { Barragán, } \\
\text { González y } \\
\text { Guzmán }\end{array}$ & México & 2017 & $\begin{array}{l}\text { Formación en TIC y competencia } \\
\text { digital en la docencia en } \\
\text { instituciones públicas de educación } \\
\text { superior }\end{array}$ & Observacional \\
\hline
\end{tabular}

Fuente: Elaboración propia

Comenzando por el contexto español, Pozo, López, Rodríguez y López (2020) llevaron a cabo con 744 profesores un estudio titulado Competencia digital docente para el uso y gestión analítica informacional del aprendizaje invertido. Este evidenció niveles intermedios de competencia digital docente: el área de mayor fortaleza fue la comunicación y la colaboración, mientras que las más débiles fueron la creación de contenidos digitales y la resolución de problemas. El estudio constató que los docentes con menores competencias evitan introducir modificaciones en su práctica, como el aula invertida o el uso del big data. Algunos de estos autores, además, publicaron el artículo Efecto de la competencia digital docente en el uso del blended learning en formación profesional, con una muestra de 627 profesores. En este quedó en evidencia el déficit en las competencias digitales de los docentes. Igual que en el estudio anterior, 


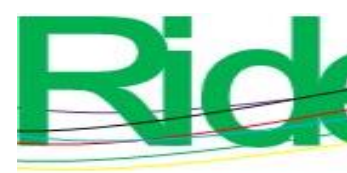

Revista Iberoamericana para la

Investigación y el Desarrollo Educativo

ISSN 2007-7467

identificaron que se evitan procesos innovadores en el aprendizaje, como el aula invertida (López, Moreno, Pozo y López, 2020).

En la misma línea, Romero, Contreras y Pérez (2019) desarrollaron un estudio titulado Las competencias mediáticas de profesores y estudiantes universitarios. Comparación de niveles en España, Portugal, Brasil y Venezuela, con 524 docentes y 1676 estudiantes universitarios de los referidos países. La investigación demostró que los dos grupos de participantes tenían un bajo nivel de competencia mediática, entendida como la capacidad de emplear de manera crítica y reflexiva la tecnología, así como las herramientas y la información disponible en línea.

Por su parte, Mirete (2016) realizó un estudio titulado El profesorado universitario y las TIC. Análisis de su competencia digital, en el que colaboraron 50 docentes de Ciencias Sociales y Jurídicas de la Universidad de Murcia. Según este trabajo, los participantes mantenían una actitud positiva hacia el uso de las TIC en la labor docente, aunque era básico el conocimiento que tenían sobre software, buscadores de información y correo electrónico.

Igualmente, en las Facultades de Ciencias Económicas y Empresariales y de Estudios Empresariales y Turismo de la Universidad de Extremadura (España) se efectuó una investigación con 84 profesores titulada La evaluación de la competencia digital en la docencia universitaria: el caso de los grados de empresariales y económicas, para conocer la percepción de docentes sobre el uso de las TIC en el ámbito profesional (Fernández, Sánchez-Oro y Robina, 2016). En este trabajo se concluye que un alto porcentaje de docentes considera esta competencia como muy importante en el desarrollo de sus asignaturas. De hecho, los participantes utilizan recursos TIC en sus materias para fomentarla y proponen actividades a través de herramientas disponibles en el campus virtual de la institución.

Ahora bien, en el contexto latinoamericano, se encontró un par de estudios llevados a cabo en Ecuador. El primero de ellos, desarrollado por Sarango-Lapo, Mena, Ramírez-Montoya y Real (2020), lleva por título La escala de competencia digital y uso de recursos educativos abiertos (CD-REA): factores asociados a la competencia de los docentes universitarios bimodales. Este se centra en 271 profesores que imparten docencia de manera presencial y a distancia. La investigación pudo confirmar un vínculo entre las competencias digitales del profesorado y las siguientes variables: años de docencia (a menor antigüedad, mayor competencia), nivel académico (maestría o doctorado con mejor competencia), número de cursos realizados (mayor capacitación es sinónimo de mayor competencia) horas de uso de internet en general (a mayor tiempo en internet, mejores habilidades), horas para comunicación con estudiantes (mejor comunicación equivale a 


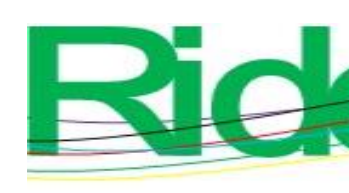

Revista Iberoamericana para la Investigación y el Desarrollo Educativo ISSN 2007-7467

mejor competencia), habilidades que posee en el uso de las TIC (mayor habilidad, mejor competencia) y número de acciones ejercidas para innovar en el aula (más acciones ejercidas equivalen a una mayor competencia).

El segundo estudio, llevado a cabo por Cejas, Lozada, Urrego, Mendoza y Rivas (2020), se denominó La irrupción de las tecnologías de la información y la comunicación (TIC): un reto en la gestión de las competencias digitales de los profesores universitarios en el Ecuador. Con una muestra de 37 profesores, los autores concluyeron que los participantes utilizan las TIC solo para la información y la comunicación, ya que existe una alta deficiencia en la capacitación de investigación, a pesar de que la institución ofrece acceso a la tecnología.

Siguiendo la misma línea, pero ahora en Colombia, García, Villareal, Cuéllar, Echeverri, Henao y Botero (2020) desarrollaron un estudio llamado Competencia digital en docentes universitarios: evaluación de relación entre actitud, formación y alfabetización en el uso de TIC en entornos educativos. Mediante un análisis de ecuaciones estructurales en una muestra de 93 docentes, los autores concluyeron que la alfabetización, la formación y la actitud hacia las TIC influyen en el uso de estas dentro del aula.

En este mismo país, Montoya y González (2019) llevaron a cabo una investigación titulada Competencias TIC en docentes de nivel técnico y tecnológico. Un estudio de caso en un centro de formación del SENA, que les permitió concluir que las diversas competencias digitales se encuentran vinculadas entre sí. Además, se encontró una correlación entre la competencia en TIC y las actitudes del profesorado. Sin embargo, aunque la mayoría tiene actitud positiva, cerca de 30 $\%$ no las considera necesarias.

También en Colombia se realizó un trabajo titulado Índice de competencias TIC en docentes de educación superior. Los resultados indican que los profesores tienen un buen manejo de los recursos tecnológicos, aunque en la competencia pedagógica se aprecia un índice bajo, por lo que el autor de la investigación sugiere que los programas de formación docente en competencias TIC, además de la parte instrumental, deben tener un fundamento epistemológico en modelos educativos (Tobar, 2017).

Por otra parte, en Chile, Solís de Ovando y Jara (2019) desarrollaron un estudio denominado Competencia digital de docentes de ciencias de la salud de una universidad chilena, con una muestra de 94 docentes de las carreras de enfermería, nutrición y dietética. Los hallazgos de la investigación destacan que el área de mayor fortaleza fue el uso técnico, mientras que el diseño de materiales fue la más débil. También constataron una correlación inversa entre las competencias 


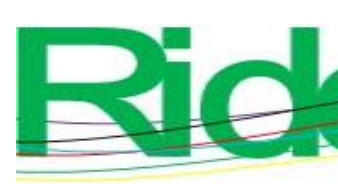

Revista Iberoamericana para la Investigación y el Desarrollo Educativo ISSN $2007-7467$

digitales y la edad (es decir, a mayor edad del profesorado, menor competencia). Del mismo modo, se constató un vínculo con los años de experiencia en la dimensión de uso didáctico. En cuestión de género, los hombres mostraron niveles superiores de competencia, mientras que el grado académico no tuvo influencia en ninguna dimensión.

Otro estudio llevado a cabo en Chile por Ríos, Gómez y Rojas (2018), titulado Valoración de competencias tic del profesorado universitario: un caso en Chile, encontró que la importancia que los docentes dan a las TIC es mayor que el dominio que tienen sobre estas. Además, se destaca que el profesorado considera que domina más los aspectos tecnológicos de su uso que los pedagógicos. El estudio descartó una relación con el género, la edad y el tipo de facultad del docente.

A continuación, en República Dominicana, se encontró una investigación desarrollada por Pérez (2019) titulada Competencia digital docente en los institutos superiores de formación de maestros: caso de República Dominicana. En una muestra de 121 profesores universitarios detectaron que el manejo de los procesadores de texto, así como el acceso, la navegación y la búsqueda en internet son las dimensiones con mejor nivel de competencia, mientras que creación de contenido digital es significativamente la más débil. Además, descartaron que la edad del profesorado influya de forma significativa en su competencia digital.

En Venezuela, Sandia, Aguilar y Luzardo (2018) llevaron a cabo un estudio titulado Competencias digitales de los docentes de educación superior. Caso Universidad de Los Andes. Con un total de 116 profesores de dos facultades distintas (Ingeniería y Ciencias Económicas y Sociales), la investigación concluyó que, en promedio, se tiene un nivel integrado (intermedio) de dominio de las TIC. Además, se encontraron diferencias de acuerdo con la facultad de adscripción, el tipo de contrato y el nivel de estudios, aunque se rechazó la influencia de la edad o el género en el nivel de competencia digital.

En este mismo país, Zambrano, Marquina, Araque y Mousalli (2016) realizaron un trabajo llamado Escuela de educación y la competencia digital de sus estudiantes y docentes, el cual fue desarrollado para conocer la competencia digital de los docentes y los estudiantes de la Escuela de Educación de la Facultad de Humanidades y Educación de la Universidad de Los Andes. Los resultados demuestran que los docentes utilizan en promedio 7 horas a la semana para planificar sus clases (75 \% utiliza internet desde sus casas). Además, tienen un mayor nivel en la dimensión aprendizaje y creatividad, pues la fomentan con mayor frecuencia, aunque no sucede lo mismo con 
el resto de las dimensiones. De acuerdo con los autores, los docentes deben ser protagonistas y buscar la integración de las TIC para aprovechar sus ventajas.

En Cuba, una investigación realizada por Avello, López y Vázquez (2016), titulada Competencias TIC de los docentes de las escuelas de Hotelería y Turismo cubanas, se enfocó en determinar las necesidades de formación de los docentes en relación con el marco de competencias TIC. El trabajo concluyó (respecto a la dimensión tecnológica) que 50 \% de los docentes no utilizan herramientas de la Web 2.0 debido, quizás, a las restricciones de acceso presentes en las escuelas. Aun así, cabe destacar el alto porcentaje de docentes que maneja equipos y redes, gestiona dispositivos y maneja paquetes de ofimática, lo cual está relacionado con la cantidad de cursos de introducción a la informática ofrecidos en la misma institución. Asimismo, un elemento evaluado positivamente fue el tema de la búsqueda y organización de la información. Con respecto a la dimensión pedagógica, $86 \%$ aplica en el aula nuevas estrategias didácticas que permiten aprovechar los recursos TIC. En cuanto a la dimensión desarrollo investigativo y profesional, se apreció que la mayoría no crea ni participa activamente en redes virtuales de investigación.

Ya en el contexto mexicano, Zárate, Gurieva y Jiménez (2020), en su estudio La práctica holística de las competencias digitales docentes: diagnóstico y prospectiva, analizaron la temática en una muestra de 18 profesores. Los resultados identifican que el uso de herramientas y la creación de contenidos son las competencias digitales más consolidadas, mientras que la colaboración representa el punto más débil. A partir de los resultados, los autores realizan un plan de mejora mediante el diseño de proyectos vinculados a las problemáticas específicas que enfrenta el estudiante.

Por otro lado, Araiza y Pedraza (2019) publicaron un estudio denominado Discernimiento de los docentes por género en el uso de las TIC en el aula a partir de las competencias digitales. En una muestra de 224 docentes, se encontraron diferencias significativas entre los docentes, pues las mujeres valoran en mayor medida la importancia del uso de las TIC en el aula, de ahí que las empleen e integren de forma más frecuente.

Finalmente, Zempoalteca, Barragán, González y Guzmán (2017) en su estudio Formación en TIC y competencia digital en la docencia en instituciones públicas de educación superior, llevado a cabo con 100 docentes, encontraron que existe relación entre el grado de competencia digital y la formación en TIC, es decir, que cuanto más se capacite el profesor, tendrá un mejor dominio. Lo anterior influye en el rendimiento académico de los estudiantes y evidencia que el uso innovador de las TIC tiene efecto favorable en la práctica académica de los alumnos. 


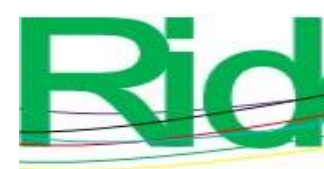

Revista Iberoamericana para la Investigación y el Desarrollo Educativo ISSN $2007-7467$

\section{Conclusiones}

En el presente estudio se concluye que en Iberoamérica, en general, los estudios se han enfocado más en el componente tecnológico de las TIC que en los aspectos de índole pedagógica. Sin embargo, se debe advertir que el simple empleo de las TIC en el proceso de enseñanzaaprendizaje es insuficiente para fomentar cambios radicales en la educación formal, ya que lo más significativo es habilitar a los docentes en el empleo de competencias digitales que potencien el aprendizaje real de los estudiantes.

Por ello, para futuros trabajos se recomienda — además de realizar más indagatorias que aborden el nivel de competencias digitales en profesores universitarios - profundizar en el impacto que estas tienen en la calidad de la docencia, y especialmente en el aprovechamiento escolar de los estudiantes.

Asimismo, se podría considerar la posibilidad de desarrollar líneas de investigación que incluyan intervenciones educativas para incrementar la habilitación en competencias digitales de los docentes universitarios. Igualmente, convendría emprender estudios de corte cualitativo para analizar los significados que los docentes han construido hacia el empleo de herramientas digitales y reconocer las motivaciones reales que tienen hacia su empleo o posible rechazo. 


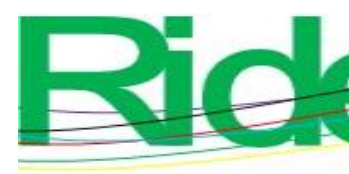

Revista Iberoamericana para la Investigación y el Desarrollo Educativo ISSN 2007-7467

\section{Referencias}

Agreda, M., Hinojo, M. y Sola, M. (2016). Diseño y validación de un instrumento para evaluar la competencia digital de los docentes en la educación superior española. Píxel-Bit. Revista de Medios y Educación, 3(49), 39-56. Recuperado de https://recyt.fecyt.es/index.php/pixel/article/view/61713

Araiza, M. y Pedraza, E. (2019). Discernimiento de los docentes por género en el uso de las TIC en el aula a partir de las competencias digitales. Revista Espacios, 40(21). Recuperado de http://www.revistaespacios.com/a19v40n21/a19v40n21p21.pdf

Avello Martínez, R., López Fernández, R. y Vázquez Cedeño, S. (2016). Competencias TIC de los docentes de las escuelas de Hotelería y Turismo cubanas. Universidad y Sociedad, 8(1). Recuperado de https://rus.ucf.edu.cu/index.php/rus/article/view/306

Castellanos, A., Sánchez, C. y Calderero, J. (2017). Nuevos modelos tecnopedagógicos. Competencia digital de los alumnos universitarios. REDIE. Revista Electrónica de Investigación Educativa, 19(1), 1-9. Doi: doi:10.24320/redie.2017.19.1.1148

Cejas, M., Lozada, B., Urrego, A., Mendoza, D. y Rivas, G. (2020). La irrupción de las tecnologías de la información y la comunicación (TIC): un reto en la gestión de las competencias digitales de los profesores universitarios en el Ecuador. RISTI. Revista lbérica de Sistemas y Tecnologías de Información, (37), 132-148. Doi: doi:10.17013/risti.37.131-148

Chancusig, J., Flores, G. y Constante, M. (2017). Las Tic's en la formación de docentes. Revista Redipe, 6(2), 174-198. $\quad$ Recuperado de https://revista.redipe.org/index.php/1/article/view/206

Delgado, Á., Vázquez-Cano, E., Belando, M. y López, E. (2019). Análisis bibliométrico del impacto de la investigación educativa en diversidad funcional y competencia digital: Web of Science y Scopus. Aula Abierta, 48(2), 147-156. Doi: doi:10.17811/rifie.48.2.2019.147156

Falcó, J. (2017). Evaluación de la competencia digital docente en la Comunidad Autónoma de Aragón. REDIE. Revista Electrónica de Investigación Educativa, 19(4), 74-83. Doi: doi:10.24320/redie.2017.19.4.1359

Fernández Sánchez, M., Sánchez-Oro Sánchez, M. y Robina Ramírez, R. (2016). La evaluación de la competencia digital en la docencia universitaria: el caso de los grados de empresariales y económicas. Revista Colombiana de Ciencias Sociales, 7(2), 332-348. Doi: doi: $10.21501 / 22161201.1726$ 


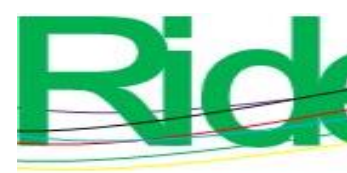

Revista Iberoamericana para la Investigación y el Desarrollo Educativo ISSN $2007-7467$

Fernández, C., Fernández, C. y Cebreiro, B. (2016). Desarrollo de un cuestionario de competencias en TIC para profesores de distintos niveles educativos. Píxel-Bit. Revista de Medios y Educación, (48), 135-148. Recuperado de https://recyt.fecyt.es/index.php/pixel/article/view/61698

García, D., Villareal, J., Cuéllar, O., Echeverri, C., Henao, C. y Botero, M. (2020). Competencia digital en docentes universitarios: evaluación de relación entre actitud, formación y alfabetización en el uso de TIC en entornos educativos. RISTI. Revista Ibérica de Sistemas e Tecnologías de Información, (E29), 538-552. Recuperado de http://www.risti.xyz/issues/ristie29.pdf

Gutiérrez, J. y Cabero, J. (2016). Estudio de caso sobre la autopercepción de la competencia digital del estudiante universitario de las titulaciones de grado de Educación Infantil y Primaria. Profesorado. Revista de Currículum y Formación del Profesorado, 20(2), 180-199. Recuperado de https://recyt.fecyt.es/index.php/profesorado/article/view/52098

Hernández, P., Gisbert, M. y Fernández, I. (2018). La evaluación de la competencia digital de los estudiantes: una revisión al caso latinoamericano. Chasqui. Revista Latinoamericana de Comunicación, (137), 91-110. Recuperado de https://dialnet.unirioja.es/servlet/articulo?codigo=6578583

López, J., Moreno, A., Pozo, S. y López, J. (2020). Efecto de la competencia digital docente en el uso del bleended learning en formación profesional. Investigación Bibliotecológica, 34(83), 187-205. Doi: doi:10.22201/iibi.24488321xe.2020.83.58147

Marqués, P. (2012). Impacto de las TIC en la educación: funciones y limitaciones. 3C TIC, Cuadernos de Desarrollo Aplicados a las TIC, 1(3), 1-15. Recuperado de https://www.3ciencias.com/wp-content/uploads/2013/01/impacto-de-las-tic.pdf

Martínez, E., Vila, E. y Gewerc, A. (2019). El papel de la familia en la construcción de la competencia digital. RISTI. Revista lbérica de Sistemas y Tecnologías de Información, 28, 1-13. Doi: doi:10.17013/risti.28.1-13

Mirete, A. B. (2016). El profesorado universitario y las TIC. Análisis de su competencia digital. Revista de la Facultad de Educación de Albacete, 31(1). Recuperado de http://www.revista.uclm.es/index.php/ensayos

Montoya, N. y González, E. (2019). Competencias TIC en docentes de nivel técnico y tecnológico. Un estudio de caso en un centro de formación del SENA. Revista Virtual Universidad Católica del Norte, (58), 74-95. Doi: doi:10.35575/rvucn.n58a3 

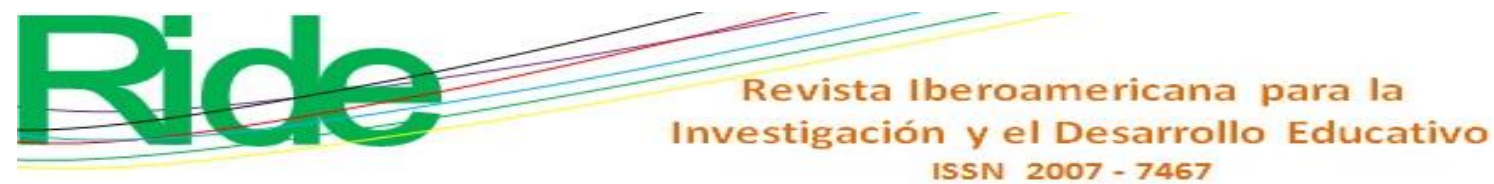

Muñoz, E. y Cubo, S. (2019). Competencia digital, formación y actitud del profesorado de educación especial hacia las tecnologías de la información y la comunicación (TIC). Profesorado. Revista de Currículum y Formación del Profesorado, 23(1), 209-241. Doi: doi:10.30827/profesorado.v23i1.9151

Padilla, A., Gámiz, V. y Romero, M. (2020). Evolución de la competencia digital docente del profesorado universitario: incidentes críticos a partir de relatos de vida. EDUCAR, 56(1), 109-127. Doi: doi:10.5565/rev/educar.1088

Perdomo, B., González O. y Barrutia, I. (2020). Competencias digitales en docentes universitarios: una revisión sistemática de la literatura. EDMETIC, Revista de Educación Mediática y TIC, 9(2), 92-115. Recuperado de https://www.uco.es/ucopress/ojs/index.php/edmetic/article/view/12796

Pérez, A. y Rodríguez, M. (2016). Evaluación de las competencias digitales autopercibidas del profesorado de Educación Primaria en Castilla y León (España). Revista de Investigación Educativa, 34(2), 399-415. Doi: doi:10.6018/rie.34.2.215121

Pérez, R. (2019). Competencia digital docente en los institutos superiores de formación de maestros: caso de República Dominicana. Pixel Bit. Revista de Medios y Educación, (55), 75-97. Doi: doi:10.12795/pixelbit.2019.i55.05

Pozo, S., López, J., Fernández, M. y López, J. (2020). Análisis correlacional de los factores incidentes en el nivel de competencia digital del profesorado. Revista Electrónica Interuniversitaria de Formación del Profesorado, 23(1), 143-159. Doi: doi:10.6018/reifop.396741

Pozo, S., López, J., Rodríguez, A. y López, J. (2020). Teachers' digital competence in using and analytically managing information in flipped learning. Culture and Education, 32(2), 1-13. Doi: doi:10.1080/11356405.2020.1741876

Regalado, J. (2013). Las competencias digitales en la formación docente. Ra Ximhai, 9(4), 21-29. Recuperado de http://www.redalyc.org/articulo.oa?id=46129004002

Reiss, C., Pessoa, T. y Gallego-Arrufat, M. (2019). Alfabetización y competencia digital en educación superior: una revisión sistemática. REDU. Revista de Docencia Universitaria, 17(1), 45-58. Doi: doi:10.4995/redu.2019.11274

Ríos, J., Gómez, E. y Rojas, M. (2018). Valoración de competencias tic del profesorado universitario: un caso en Chile. Píxel-Bit. Revista de Medios y Educación, (52), 55-65. Doi: doi:10.12795/pixelbit.2018.i52.04 


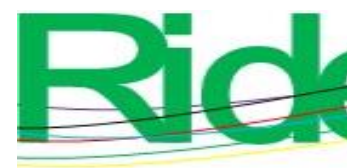

Revista Iberoamericana para la Investigación y el Desarrollo Educativo ISSN $2007-7467$

Roblizo, M., Sánchez, M. y Cózar, R. (2015). El reto de la competencia digital en los futuros docentes de infantil, primaria y secundaria. Los estudiantes de grado y máster de educación ante las TIC. Prisma Social, (15), 254-295. Recuperado de https://www.redalyc.org/articulo.oa?id=353744533008

Rodríguez, A. y Martínez, N. (2018). La competencia digital en la base de Scopus: un estudio de metaanálisis. REXE. Revista de Estudios y Experiencias en Educación, 2(2), 15-24. Recuperado de http://www.rexe.cl/ojournal/index.php/rexe/article/view/544

Rodríguez, A., Raso, F. y Ruiz, J. (2019). Competencia digital, educación superior y formación del profesorado: un estudio de meta-análisis en la Web of Science. Píxel-BIT. Revista de Medios y Educación, (54), 65-81. Doi: doi:10.12795/pixelbit.2019.i54.04

Rodríguez, A., Trujillo, J. y Sánchez, J. (2019). Impacto de la productividad científica sobre competencia digital de los futuros docentes: aproximación bibliométrica en Scopus y Web of Science. Revista Complutense de Educación, 30(2), 623-646. Doi: doi:10.5209/RCED.58862

Romero, L., Contreras, P. y Pérez, A. (2019). Las competencias mediáticas de profesores y estudiantes universitarios. Comparación de niveles en España, Portugal, Brasil y Venezuela. Cultura y Educación, 31(2), 326-368. Doi: doi:10.1080/11356405.2019.1597564

Sánchez, P., Andrés, C. y Paredes, J. (2019). El papel de la familia en el desarrollo de la competencia digital. Análisis de cuatro casos. Digital Education Review, (34), 44-58. Recuperado de https://revistes.ub.edu/index.php/der/article/view/20750/pdf

Sandia, B., Aguilar, A. y Luzardo, M. (2018). Competencias digitales de los docentes de educación superior. Caso Universidad de Los Andes. EDUCERE, 22(73), 603-616. Recuperado de https://www.redalyc.org/articulo.oa?id=35656676011

Sarango-Lapo, C., Mena, J., Ramírez-Montoya, M. y Real, E. (2020). La escala de competencia digital y uso de recursos educativos abiertos (CD-REA): factores asociados a la competencia de los docentes universitarios bimodales. RISTI. Revista Ibérica de Sistemas e Tecnologías de Información, (E-28), 544-558. Recuperado de http://www.risti.xyz/issues/ristie28.pdf

Solís de Ovando, J. y Jara, V. (2019). Competencia digital de docentes de ciencias de la salud de una universidad chilena. Pixel Bit. Revista de Medios y Educación, (56), 193-211. Doi: doi:10.12795/pixelbit.2019.i56.10 


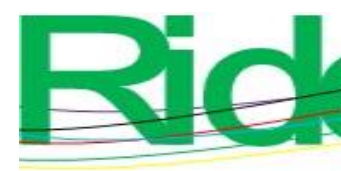

Revista Iberoamericana para la
Investigación y el Desarrollo Educativo
ISSN $2007-7467$

Suárez, J., Almerich, G., Díaz, I. y Fernández, R. (2011). Competencias del profesorado en las TIC. Influencia de factores personales y contextuales. Universitas Psychologica, 11(1), 293-309. Recuperado de http://www.redalyc.org/articulo.oa?id=64723234024

Tobar, A. O. (2017). Índice de competencias TIC en docentes de educación superior. Campus $\begin{array}{llll}\text { Virtuales, } & 6(2), & \text { 113-125. } & \text { Recuperado }\end{array}$ http://uajournals.com/ojs/index.php/campusvirtuales/article/view/240

Valerio, C. y Paredes, J. (2008). Evaluación del uso y manejo de las tecnologías de información y comunicación en los docentes universitarios. Un caso mexicano. Revista Latinoamericana de Tecnología Educativa, 7(1), 13-32. Recuperado de https://relatec.unex.es/article/view/391/321

Vázquez-Cano, E., Reyes, M., Colmenares, L. y López, E. (2017). Competencia digital del alumnado de la Universidad Católica de Santiago de Guayaquil. Opción, 33(83), 229-251. Recuperado de https://www.redalyc.org/articulo.oa?id=31053772008

Zambrano Contreras, J. C., Marquina Gutiérrez, R. A., Araque Vergara, Y. K. y Mousalli Kayat, G. (2016). Escuela de educación y la competencia digital de sus estudiantes y docentes. Revista Eduweb, 10(1), 41-53. $\quad$ Recuperado de https://revistaeduweb.org/index.php/eduweb/article/view/82

Zárate, A., Gurieva, N. y Jiménez, V. (2020). La práctica holística de las competencias digitales docentes: diagnóstico y prospectiva. Pensamiento Educativo. Revista de Investigación Educacional Latinoamericana, 57(1), 1-16. Doi: doi:10.7764/PEL.57.1.2020.10

Zempoalteca, B., Barragán, J., González, J. y Guzmán, T. (2017). Formación en TIC y competencia digital en la docencia en instituciones públicas de educación superior. Apertura, 9(1), 1-24. Doi: doi:10.32870/Ap.v9n1.922 


\begin{tabular}{|c|c|c|}
\hline Rol de Contribución & Autor (es) & \\
\hline Conceptualización & Juan Carlos Padilla Escobedo (principal) & \\
\hline Metodología & $\begin{array}{l}\text { Juan Carlos Padilla } \quad \text { Escobedo } \\
\text { Graciela Gerarda Ayala Jiménez (apoyo) }\end{array}$ & $($ principal $)$ \\
\hline Software & NO APLICA & \\
\hline Validación & $\begin{array}{l}\text { Juan Carlos } \quad \text { Padilla } \quad \text { Escobedo } \\
\text { Graciela Gerarda Ayala Jiménez (apoyo) }\end{array}$ & (principal) \\
\hline Análisis Formal & Graciela Gerarda Ayala Jiménez (principal) & \\
\hline Investigación & Juan Carlos Padilla Escobedo (principal) & \\
\hline Recursos & NO APLICA & \\
\hline Curación de datos & NO APLICA & \\
\hline $\begin{array}{l}\text { Escritura - Preparación del } \\
\text { borrador original }\end{array}$ & $\begin{array}{l}\text { Juan Carlos Padilla } \quad \text { Escobedo } \\
\text { Graciela Gerarda Ayala Jiménez (apoyo) }\end{array}$ & (principal) \\
\hline Escritura - Revisión y edición & $\begin{array}{l}\text { Juan Carlos Padilla } \quad \text { Escobedo } \\
\text { Graciela Gerarda Ayala Jiménez (apoyo) }\end{array}$ & (principal) \\
\hline Visualización & Juan Carlos Padilla Escobedo (principal) & \\
\hline Supervisión & Graciela Gerarda Ayala Jiménez (principal) & \\
\hline Administración de Proyectos & NO APLICA & \\
\hline Adquisición de fondos & NO APLICA & \\
\hline
\end{tabular}

